What's New from

Juntendo University, Tokyo

Juntendo Medical Journal

2014. $60(6), 523$

\title{
Fourth Juntendo University-Hitachi Cooperative Research Workshop
}

Development of a Non-Invasive Diagnostic Method for Esophageal Squamous Cell Carcinoma

by Gas Chromatographic Analysis of Patients' Exhaled Breath

\author{
SEI MATSUMORI*1), NAOKO KAGA*2), HikARI TAKA*2), TsUTOMU FUJIMURA*2), \\ TAKASHI UENO*2), FUYUMI ISAYAMA*1), YOSHIAKI KAJIYAMA*1)
}

*1) Department of Esophageal and Gastroenterological Surgery, Juntendo University Faculty of Medicine,Tokyo, Japan,

*2) Laboratory of Proteomics \& Biomolecular Science Biomedical Research Center, Juntendo University Faculty of Medicine,

Tokyo, Japan

Background: Since esophageal carcinoma progresses asymptomatically, for many patients, the disease is already advanced at the time of diagnosis. Compared with other digestive organ cancers, esophageal carcinoma progresses rapidly and has a poor prognosis, making early diagnosis important. There is a need to develop a diagnostic method using clinical markers that is non-invasive while being both highly sensitive and specific.

Method: Exhaled breath was collected from 17 patients with esophageal squamous cell carcinoma, as well as 9 healthy subjects (control group) with no history of cancer. For each fasting subject, $1 \mathrm{~L}$ of exhaled breath was collected in a gas sampling bag. Volatile organic compounds (VOCs) were then extracted from each sample using Solid-Phase Micro-Extraction (SPME) fibers and analyzed by gas chromatography.

Results: Comparison of the principal component analyses of the VOCs showed a significant difference between the patient group and the healthy control group. In the patient group, significant increases were observed in 4 VOCs, namely, acetonitrile, acetic acid, acetone, and 2-butanone. Receiver operating characteristic (ROC) curves were drawn for acetonitrile, acetic acid, acetone, and 2-butanone. The calculated area-under-the-curve (AUS) indicated that esophageal carcinoma patients can be identified with a high probability of 0.93 .

Conclusions: It was confirmed that there are significant differences in the contents of certain VOCs contained in the exhaled breath of esophageal carcinoma patients compared with that of healthy subjects. The analysis for these VOCs is inexpensive, simple and non-invasive, causing no adverse reactions, and it has potential as a new tool for detecting early-stage esophageal carcinoma.

Key words: breath analysis, esophageal carcinoma, gas chromatography, volatile organic compounds
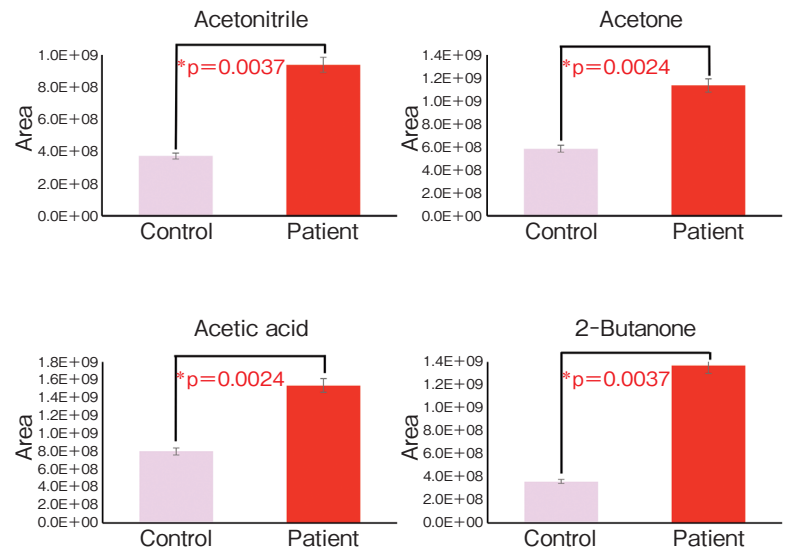

Figure-1 Breath components of patients and controls

\section{References}

1) Bijland LR, Bomers MK, Smulders YM: Smelling the diagnosis: a review on the use of scent in diagnosing disease. Neth J Med, 2013; 71: 300-307.

2) Filipiak W, Sponring A, Filipiak A, et al: TD-GC-MS analysis of volatile metabolites of human lung cancer and normal cells in vitro. Cancer Epidemiol Biomarkers Prev, 2010; 19: 182-195.

3) Kumar S, Huang J, Abbassi-Ghadi N, Hanna GB, et al: Selected ion flow tube mass spectrometry analysis of exhaled breath for volatile organic compound profiling of esophago-gastric cancer. Anal Chem, 2013; 85: 61216128.

Corresponding author: Sei Matsumori

Department of Esophageal and Gastroenterological Surgery, Juntendo University Faculty of Medicine

2-1-1 Hongo, Bunkyo-ku,Tokyo 113-8421, Japan

TEL: +81-3-3813-3111 E-mail: sematsu@juntendo.ac.jp

Fourth Juntendo University-Hitachi Cooperative Research Workshop〔Held on July 26, 2014〕

[Received Oct. 30, 2014] 